\title{
OBSERVACIONES SOBRE LOS GAMETOFITOS DE WOODWARDIA MARTINEZII MAXON EX WEATHERBY Y W. SPINULOSA MART. \& GAL. (BLECHNACEAE)
}

\author{
Blanca Perez-Gabcia y Ramon Riba \\ Universidad Autónoma Metropolitana-Iztapalapa \\ Departamento de Biología, C.B.S. \\ Apartado Postal 55-535 \\ 09340 México, D. F.
}

\begin{abstract}
RESUMEN
En este trabajo se proporciona información adicional sobre la morfogénesis de la fase gametofítica de dos especies de helechos de la familia Blechnaceae. Woodwardia spinulosa, que se encuentra desde el norte de México hasta América Central (Nicaragua), tiene esporas de color pardo oscuro, bilaterales, elipsoidales y con perispora en forma de pliegues ondulados, irregulares y evidentes; el patrón de germinación es de tipo Vittaria y el desarrollo protálico de tipo Aspidium. A la madurez, los gametofitos son bisexuados, con anteridios y arquegonios aparentemente normales del tipo común de los helechos leptosporangiados. A los 11 meses después de la germinación se observaron esporofitos juveniles con 102 hojas. W. martinezii solamente es conocida de unas cuantas localidades en los estados de Veracruz e Hidalgo, en México; sus esporas y los patrones de germinación y de desarrollo protálico son similares a los de $W$. spinulosa, aunque las esporas son de mayor tamaño. Se observaron gametofitos maduros espatulados con diferenciación de anteridios y sin rastros de arquegonios y gametofitos vegetativos, en los que hubo desarrollo de esporofitos apogámicos. El desarrollo de los gametofitos de estas dos especies concuerda con el de otros géneros de la familia Blechnaceae estudiados por otros autores.
\end{abstract}

\section{ABSTRACT}

Additional information is given in this paper on morphogenetic features of the gametophyte of two fern species of the Blechnaceae. Woodwardia spinulosa, growing from the North of Mexico to Central America (Nicaragua) has dark brown, bilateral and ellipsoidal spores with wavy and folded perispore; the germination pattern is of Vittaria type and the prothallial development is of Aspidium type. The mature gametophytes are bisexual, with antheridia and archegonia typical of leptosporangiate ferns. Juvenile sporophytes with one or two leaves were observed 11 months after spore germination. Woodwardia martinezii is only known from a few localities in the states of Veracruz and Hidalgo, Mexico; the spores and the germination and prothallial development patterns are similar to those of $W$. spinulosa, but the spores are larger. Spatulate gametophytes with antheridia and without archegonia were observed, and also vegetative gametophytes with apogamous sporophytes. The gametophyte development of these two species is consistent with other blechnaceous ferns previously studied. 


\section{INTRODUCCION}

La familia Blechnaceae está representada en América por los géneros Woodwardia, Blechnum y Salpichlaena, este último exclusivamente americano y los dos primeros con especies también en el Viejo Mundo. En términos generales sus gametofitos tienen una configuración estructural similar, son epigeos, clorofílicos, cordiformes a alargados, con cojinete central y por lo regular bisexuados. El género Woodwardia cuenta con cerca de doce especies, incluyendo un híbrido americano aparentemente estéril (Mickel \& Beitel, 1988); en México crecen W. spinulosa, W. martinezii y el híbrido W. semicordata Mickel \& Beitel.

Woodwardia spinulosa es una especie neotropical que se distribuye desde el norte de México hasta Nicaragua y en este trabajo se acepta como distinta de $W$. fimbriata, de la cual la consideran sinonimo Tryon y Tryon (1982); $W$. spinulosa se diferencia por las escamas fibrilosas en el lado abaxial de la costa, venas y lámina, por carecer de glándulas de resina de color amarillo pálido en el envés y por la base cortamente decurrente de las pinnas superiores, la que en $W$. fimbriata es cordiforme. W. martinezii es una especie mexicana de distribución muy restringida, pues solamente se conoce de unas pocas localidades cercanas entre si de los estados de Hidalgo (Tlanchinol y Tianguistengo) y Veracruz (Huayacocotla).

Estudios previos (Nayar et al., 1966; Stone 1961, 1969) muestran que los gametofitos de algunas especies australianas de Blechnaceae pueden distinguirse entre si por sus rasgos morfológicos (desarrollo de pelos y tipo de margen) pero, en general, aunque hay diferencias menores entre los gametofitos de las especies estudiadas, éstas no son más significativas para los géneros de la familia Blechnaceae que para las especies de los mismos géneros.

El resultado de nuestras observaciones agrega información a la ya existente referente a la morfogénesis de la fase gametofítica de especies de la familia Blechnaceae.

\section{MATERIAL Y METODO}

Los ejemplares fértiles de Woodwardia spinulosa fueron recolectados en el estado de Hidalgo, México, en los alrededores de Zacualtipán a $2000 \mathrm{~ms}$.n.m. en bosque mixto de pinoencino (B. Pérez-Garcla 987, UAMIZ). El material fértil de Woodwardia martineziise recolectó en bosque mesófilo de montaña muy alterado, en el km 147 de la carretera México-Tampico, cerca de Tlanchinol, Hidalgo, México a 1640 m s.n.m.(R. Riba 1848, UAMIZ). Las esporas de ambas especies se obtuvieron guardando segmentos fértiles con esporangios maduros y cerrados en sobres de papel, dejándolos secar a temperatura ambiente para propiciar su apertura por desecación en forma natural; el material asi obtenido se tamizó para eliminar fragmentos de esporangios y otras impurezas.

Se sembraron esporas de cada especie con una densidad media de 1000 esporas por $\mathrm{cm}^{2}$ en medio de Thompson con agar (Pérez-García, 1989) en cinco cajas de Petri, una de las cuales se cubrí con papel estaño para probar fotoblastismo. Los cultivos se mantuvieron en un régimen lumínico de $12 \times 12$ horas, a una temperatura de $20-28^{\circ} \mathrm{C}$. Las cinco cajas se mantuvieron dentro de bolsas de polietileno transparente durante todo el proceso, abriéndose solamente cada 8-10 días durante las revisiones periódicas para definir el inicio de la germinación; en cada revisión se humedeció el medio con unas cuantas gotas de agua destilada previamente esterilizada, con objeto de evitar la desecación y de propiciar el 
desplazamiento de los anterozoides, cuando los gametofitos alcanzaran la madurez sexual; la caja mantenida en la oscuridad se abrí hasta los 100 días después de la siembra.

Los dibujos se hicieron mediante el uso de una cámara clara AO y las fotografías se tomaron con un fotomicroscopio Zeiss y película Plus- $X$ de $35 \mathrm{~mm}$.

\section{RESULTADOS}

Esporas. Las esporas de Woodwardia spinulosay de W. martinezii son de color pardo oscuro, bilaterales y elipsoidales, con perispora evidente como largos pliegues prominentes e irregulares; $W$. spinulosatiene 64 esporas por esporangio, miden $37 \times 55 \mu \mathrm{m}$ y $W$. martinezii tiene 32 esporas por esporangio, miden $50 \times 74 \mu \mathrm{m}$ (incluyendo la perispora en los dos casos).

Germinación. En ambas especies, en los cultivos expuestos a la luz, la germinación es de tipo Vittaria, se inicia entre 4-6 días después de la siembra y subsecuentemente se forma un filamento de 2-4 células con un rizoide perpendicular originado de la célula basal. Las esporas mantenidas en la oscuridad no mostraron señales de germinación después de 100 días de sembradas.

Fase laminar. En las dos especies, a los 8 días cambia la orientación del plano de división de las células apicales y se inicia la formación del incipiente gametofito laminar; este proceso continúa y a los 20-25 días el gametofito mide 0.4-0.5 $\times$ 0.15-0.20 mm. Algunas de las células apicales forman precozmente pequeños pelos secretores (Fig. 1) con cloroplastos, el desarrollo protálico es de tipo Aspidium; en esta etapa se diferencia la zona meristemática pluricelular, de posición central apical entre dos alas irregulares incipientes (Fig. 2); el margen es irregular por las proyecciones celulares terminadas en pelos cortos (Fig. 3); pelos de este mismo tipo aparecen a los 25 días en ambas caras del gametofito laminar (Fig. 4).

Gametofitos adultos. A los 30 días, los gametofitos de W. martinezii son espatulados alargados a acintados o cordiformes, pero irregulares por el crecimiento asincrónico de los márgenes y de las proyecciones marginales pluricelulares (Fig. 5). La parte central de la lámina es más gruesa por el desarrollo de un cojinete, con numerosos rizoides de color pardo claro a pardo oscuro a todo lo largo de su cara inferior con algunos rizoides ocasionales en la cara superior.

El gametofito de $W$. spinulosa es un tanto más típico y a los 50-60 días tiende a ser cordiforme a espatulado, con los márgenes más regulares y con un pelo corto en algunas de las células marginales. Cuando los gametofitos crecen están muy aglomerados tienden a adquirir una forma acintada, crecen erectos y en ellos se diferencia gran cantidad de anteridios típicos en ambas caras y en sus márgenes. Los rizoides tienen la misma disposición que en W. martinezii.

Gametangios. En los gametofitos de W. spinulosa, entre los 3-5 meses se diferencian abundantes anteridios de posición marginal en el tercio basal (en donde por lo general se encuentra todavía la cubierta de la espora) y superficiales en la cara inferior de la lámina. Están formados por una célula basal, una anular y una opercular discoidal convexa y de menor diámetro que la célula anular. Los arquegonios se diferencian a los 3-4 meses (Fig. 8); se 

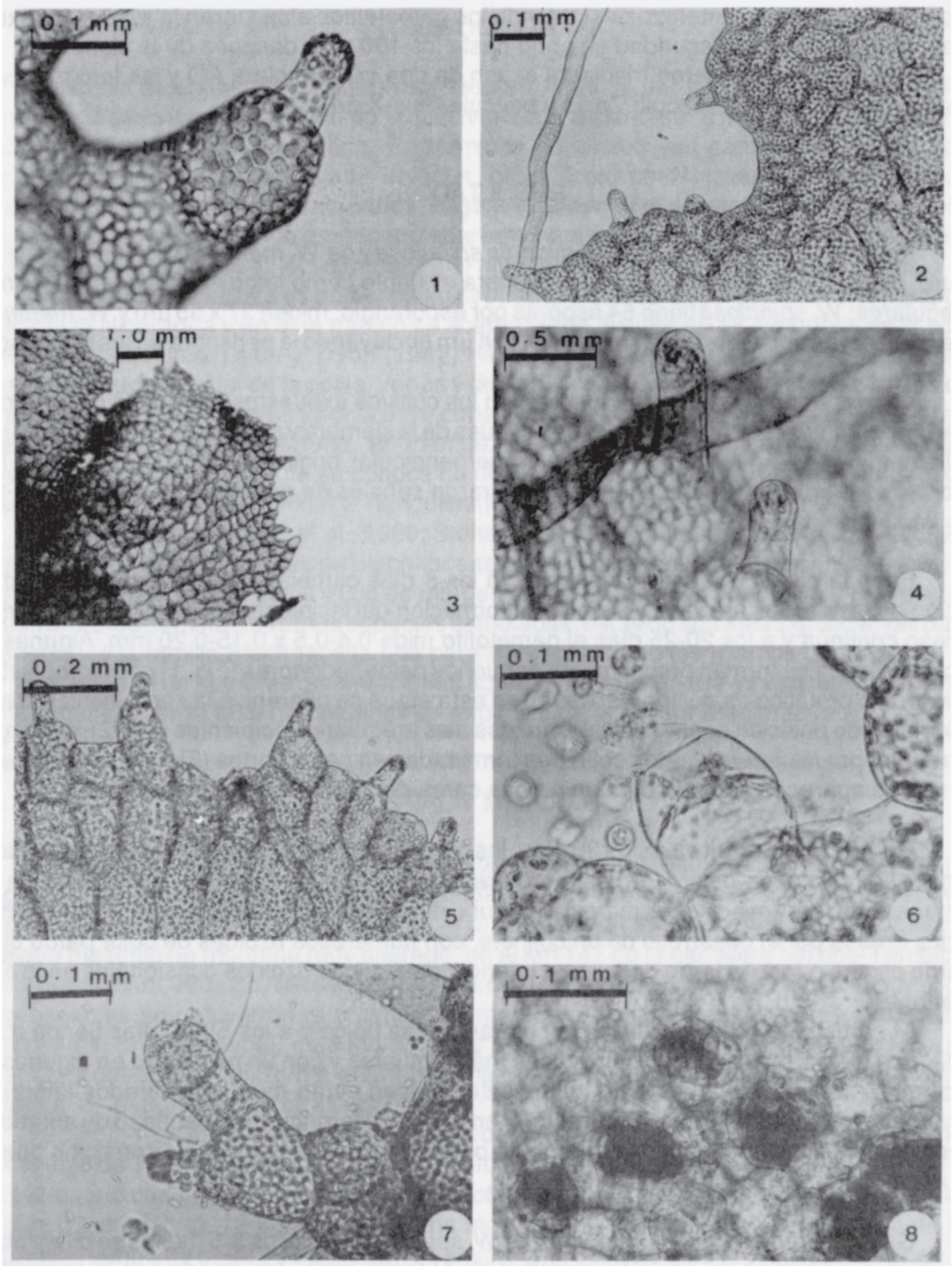

Figs. 1-7. Gametofitos de W. martinezii. 1. Pelo secretor marginal; 2. Zona meristemática; 3. Margen irregular con derivados; 4 . Pelos marginales; 5 . Margen con pelos secretores; 6 . Anteridio superficial abierto; 7. Anteridio marginal terminal. Fig. 8. Arquegonios de W. spinulosa. 
encuentran principalmente cerca de la región meristemática con los cuellos emergentes ligeramente orientados en sentido opuesto a la misma. W. martineziitiene gametofitos de dos tipos: unos son alargados, de forma irregular, algo sinuosos y a los cinco meses miden $6 \times 4$ $\mathrm{mm}$; se caracterizan por el desarrollo de numerosos anteridios (Figs. 6 y 7), similares a los de $W$. spinulosay de posición marginal y superficial, pero carentes de arquegonios. En el otro tipo, los gametofitos tienden a ser cordiformes aunque de contorno irregular y en ellos no se diferencian anteridios ni arquegonios. En ambas variantes las células vegetativas de la lámina son isodiamétricas sin diferenciación alguna, excepto las marginales, que tienden a ser alargadas, paralelas al borde.

Esporofitos. A los 11 meses después de la germinación se observaron esporofitos jóvenes con 162 hojas en los cultivos de W. spinulosa (Fig. 10). En los cultivos de W. martinezii a los 68 días se aprecia en el cojinete un crecimiento alargado que se diferencia en su extremo anterior en un primordio foliar (Fig. 14); éste se alarga y se ensancha en su ápice en la lámina de la primera hoja que es entera, de forma ovada a cortamente espatulada al principio y suavemente lobada al terminar su diferenciación (Fig. 11); el peciolo y la lámina (Figs. 13 y 15) tienen pelos de dos tipos, unos unicelulares y semejantes a los del gametofito y otros de 2 a varias células cortas, ramificados irregularmente con una célula terminal roma y aparentemente glandulosa, de color pardo. En el peciolo de la primera hoja del esporofito es posible observar por transparencia traqueidas anilladas a escalariformes formando un incipiente cordón vascular de 1-3 traqueidas de grosor que corre desde la raíz primaria hasta cerca del ápice de la hoja (Fig. 9). Este cordón vascular es simple en el primordio foliar y es una o dos veces bifurcado en la lámina de la primera hoja. Las células de la epidermis son ligeramente alargadas en el sentido de la longitud de la hoja y de contorno suavemente ondulado. Los estomas solamente se observan en la cara abaxial de la hoja. Estos últimos, asi como las células epidérmicas de las hojas son similares en $W$. spinulosa y en $W$. martinezii (Figs. 12 y 16).

\section{DISCUSION}

En términos generales los gametofitos de Woodwardia martinezii y de $W$. spinulosa tienen el mismo patrón de desarrollo de los helechos leptosporangiados y, en particular, son semejantes a los de otras especies de Blechnaceae (Nayar et al., 1966; Stone, 1969). El desarrollo del gametofito de W. spinulosa no habla sido estudiado hasta ahora; Stone (1969) y Nayar et al. (1966) investigaron el desarrollo del gametofito de W. martinezii, pero no dan la localidad original de procedencia de su material, y en ambas publicaciones indican que éste les fue enviado de Royal Botanical Gardens de Kew (Stone señala "N. America"). Nuestras plantas proceden de poblaciones silvestres del estado de Hidalgo, México.

Gametofitos. En este estudio, la germinación de las esporas de ambas especies se inició a los 4-6 días después de la siembra; Nayar et al. (1966) y Stone (1969) registran 5-7 días, 2-3 días y aun 8-10 días para el inicio de la germinación pero este lapso es variable, ya que una diferencia de $2-3^{\circ} \mathrm{C}$ en la temperatura de incubación o ligeras variantes en la iluminación (distancia-intensidad) pueden retrasar o acelerar el proceso aun para una misma especie (Pérez-García y Riba, 1982), por lo que no hay diferencia significativa con nuestros resultados. El porcentaje de germinación alcanzado fue de $100 \%$ para las dos especies después de 30 días. 

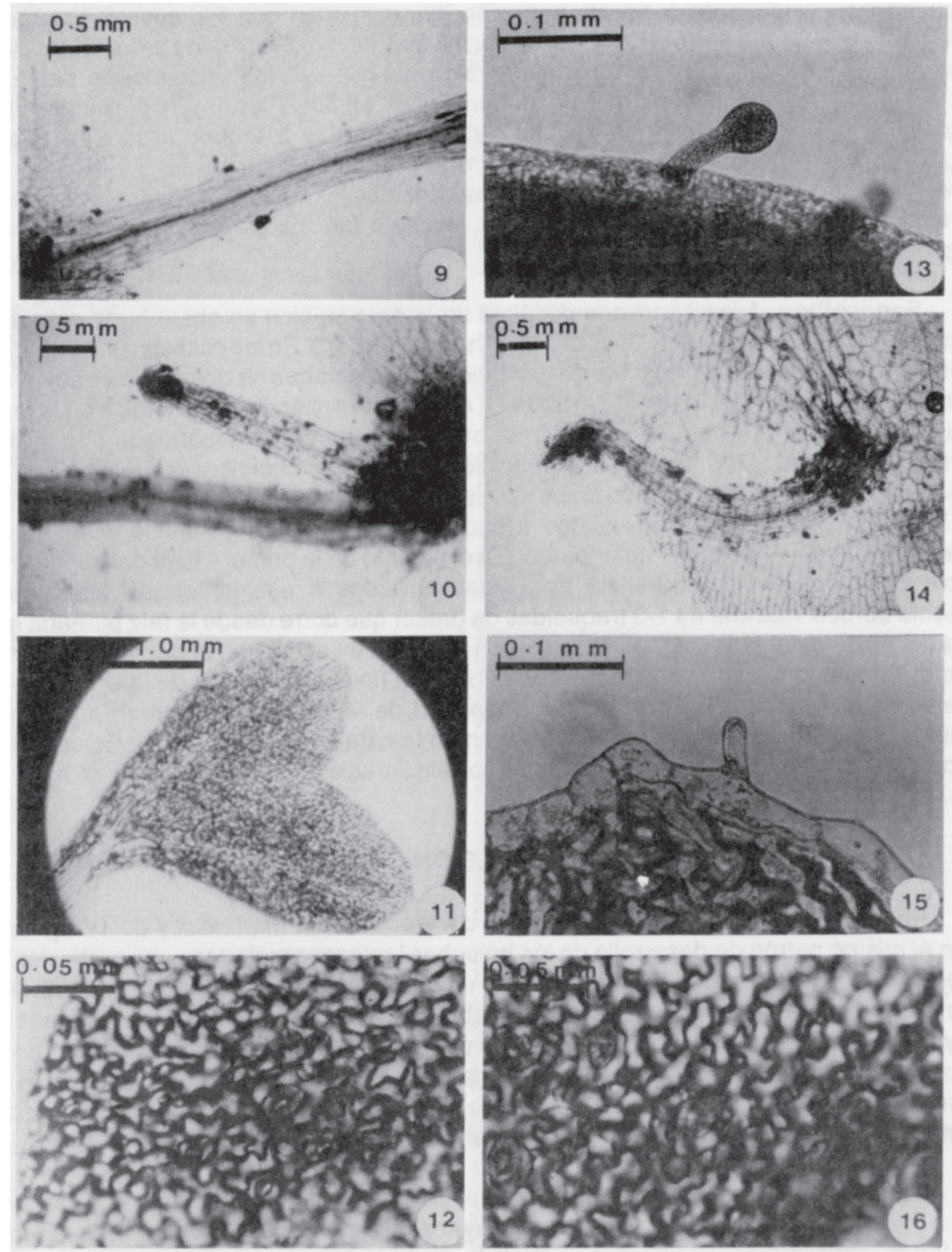

Figs 9-12. Esporofitos de W. spinulosa. Figs. 13-16. Esporofitos de W. martinezii. 9. Peciolo con cordón vascular central; 10. Primera (al fondo) y segunda hoja; 11. Lámina de la primera hoja; 12. Estomas; 13. Pelo secretor del pecíolo; 14. Esporofito apogámico joven; 15. Borde de la primera hoja con pelo secretor; 16. Estomas. 
En el filamento primario de $W$. spinulosa (2-4 células) frecuentemente la célula basal es la única que permanece indivisa y las subsecuentes se dividen pronto y desaparece el filamento como tal iniciándose tempranamente el crecimiento bidimensional de manera regular. En W. martinezii esto es menos frecuente, el filamento central desaparece pronto ya que se provoca el desplazamiento lateral de una de las células hijas de la célula basal con sus células hijas derivadas, como resultado del crecimiento y división de la otra célula que es la responsable de la formación de la zona meristemática, la que ocupará después la típica posición central.

Los gametofitos adultos en ambas especies son similares a los descritos por Stone y Nayar et al. y no adquieren la típica forma acorazonada de las especies de Doodia, Blechnum y de otros helechos blecnáceos y de muchos otros helechos leptosporangiados que comúnmente es reproducida en los libros de botánica. El margen es muy irregular a causa de las proyecciones pluricelulares laterales que, por lo general, terminan en un pelo apical.

Los gametofitos de $W$. spinulosa son bisexuados y a pesar de que se observaron anterozoides libres desplazándose en el medio, no hubo indicios de fecundación a los 150 días de la siembra de las esporas (véase adelante: Esporofitos); los anteridios tienen la constitución típica de aquellos de los helechos leptosporangiados, con una célula basal, una anular y una opercular. Los arquegonios se localizan en la región posterior a la zona meristemática, el cuello sobresale orientado hacia la región posterior del gametofito adulto, sin características distintivas definidas. W. martineziiforma gametofitos de dos tipos, aunque no hay diferencia en el tamaño y forma de las esporas; en un tipo se forman abundantes anteridios en ambas caras y en los márgenes, con diferenciación de anterozoides aparentemente normales mientras que el otro tipo es vegetativo y no se observan indicios de diferenciación de gametangios.

Esporofitos. Los gametofitos de W. spinulosa formaron esporofitos juveniles con 16 2 hojas, 11 meses después de la germinación de las esporas. En los gametofitos vegetativos de $W$. martinezii se diferencian esporofitos apogámicos a partir de un engrosamiento en la parte ventral de la zona media, que fueron evidentes a los 68 días a partir de la siembra; este mismo hecho lo observó Stone (1969) pero no es mencionado por Nayar et al. (1966). La primera hoja del esporofito de las dos especies estudiadas tiene pecíolo corto y lámina pequeña ovada a cortamente espatulada, similar a la primera hoja de muchas especies de helechos leptosporangiados. Los tricomas del peciolo son de dos tipos, pues además de aquellos similares a los del gametofito existen otros, formados por células cortas y con una ramificación en la célula basal o en la segunda. La lámina no presenta rasgos distintivos, tiene pelos marginales claviformes, epidermis con células de contorno ligeramente sinuado, estomas en la cara abaxial y mesófilo no diferenciado. La vascularización está constituida por un solo haz de 1-3 traqueidas de grosor, que se extiende desde la raiz hasta el peciolo y se continúa hasta la lámina en donde se ramifica pseudodicotómicamente una vez; las traqueidas, aunque no del todo diferenciadas, son visibles con facilidad en material transparentado.

En resumen, el desarrollo y las características morfológicas de los gametofitos de las dos especies de Woodwardia estudiadas en este trabajo, son consistentes con los de otros helechos blecnáceos estudiados por Nayar et al. y por Stone, sin peculiaridades que los distingan de otras especies. Aunque el porcentaje de germinación de las esporas de $W$. martinezii es alto, su distribución restringida indica que probablemente es una especie apogámica de reciente aparición. 


\section{LITERATURA CITADA}

Mickel, J. T. \& J. M. Beitel. 1988. Pteridophyte flora of Oaxaca, Mexico. Mem. New York Bot. Gard. 46: 1-568. Nayar, B. K., N. Bajpai \& F. Raza. 1966. Morphological studies on some species of Blechnum, Doodia, Woodwardiaand Stenochlaena: 1. The gametophytes and the juvenile sporophytes. J. Linn. Soc., Bot. 59: 405-423.

Nayar, B. K. \& S. Kaur. 1971. Gametophytes of homosporous ferns. Bot. Rev. (Lancaster) 37(3): 353.

Pérez-García, B. 1989. Morfogénesis de gametofitos de helechos arborescentes (Cyatheaceae). Tesis Doctoral. Facultad de Ciencias, Universidad Nacional Autónoma de México. México. 224 pp.

Pérez-García, B. \& R. Riba. 1982. Germinación de esporas de helechos arborescentes (Cyatheaceae) bajo diferentes condiciones de temperatura. Biotropica 14: 281-287.

Stone, I. G. 1961. The gametophytes of Victorian Blechnaceae. I. Blechnum nudum (Labill.) Luerss. Austral. J. Bot. 9: 20-36.

Stone, I. G. 1969. The gametophytes of the Victorian Blechnaceae. II. Doodia aspera R. Br., D. media R. Br. \& D. caudata $\mathrm{R}$. Br.: a comparison with three extra-Australian genera, Brainea, Sadleria and Woodwardia. Austral. J. Bot. 17: 31- 57.

Tryon, R. M. \& A. F. Tryon. 1982. Ferns and allied plants with special reference to tropical America. SpringerVerlag, New York. 662 pp. 